\title{
Is gestational diabetes an independent risk factor of neonatal severe respiratory distress syndrome after 34 weeks of gestation? A prospective study
}

\author{
Isabelle Mortier ${ }^{1}$. Julie Blanc ${ }^{1,2} \cdot$ Barthelemy Tosello $^{3,4} \cdot$ Catherine Gire $^{3}$. \\ Florence Bretelle $^{1,4} \cdot$ Xavier Carcopino ${ }^{1,5}$
}

\begin{abstract}
Purpose To evaluate if neonates delivered after $34^{0 / 7}$ weeks from mothers diagnosed with gestational diabetes (GD) are exposed to an increased risk of neonatal severe respiratory distress syndrome (SRDS).

Methods Women with singleton pregnancy in labour after $34^{0 / 7}$ weeks of gestation or admitted for planned caesarean section and who had been systematically screened for GD were eligible to participate to this prospective cohort study. Diagnosis of SRDS was defined by the association of clinical signs of early neonatal respiratory distress, with consistent radiologic features and requiring mechanical ventilation with a fraction of inspired oxygen $\left(\mathrm{FiO}_{2}\right)>0.25$ for a minimum of $24 \mathrm{~h}$ and admission to neonatal intensive care unit. Results A total of 444 women were included. GD was diagnosed in 60 patients (13.5\%). A neonatal SRDS was diagnosed in 32 cases ( $7.2 \%$ ). Compared to others, neonatal SRDS was significantly more often observed in neonates from women diagnosed with GD: 12 (20\%) vs. 20 (5.2\%),
\end{abstract}

Julie Blanc

julievirginie.blanc@ap-hm.fr

1 Department of Obstetrics and Gynaecology, Hôpital Nord, APHM, chemin des Bourrely, 13015 Marseille, France

2 EA 3279, Public Health, Chronic Diseases and Quality of Life, Research Unit, Aix-Marseille University, 13284 Marseille, France

3 Department of Neonatology, Hôpital Nord, APHM, chemin des Bourrely, 13015 Marseille, France

4 URMITE, Aix-Marseille University (AMU), UM 63, CNRS 7278, IRD 198, INSERM 1095, Institut Hospitalo-Univ ersitaire-Méditerranée Infection, 19-21 Bd Jean Moulin, 13385 Marseille Cedex 05, France

5 Aix-Marseille University (AMU), CNRS, IRD IMBE UMR 7263, 13397 Marseille, France respectively $(p<0.001)$. Women whose neonates presented neonatal SRDS were significantly more likely to be obese $(p=0.002)$, to have undergone a caesarean section $(p<0.001)$ and to have received corticosteroids therapy before $34^{0 / 7}$ weeks $(p=0.013)$. In multivariate analysis, GD was identified as an independent risk factor of neonatal SRDS (aOR 3.6; 95\% CI 1.5-8.6; $p=0.005$ ). Other risk factors were maternal obesity (aOR 2.8 ; 95\% CI 1.1-7.1; $p=0.029$ ) and assisted vaginal delivery (aOR 5.5; 95\% CI $1.9-15.9 ; p=0.002$ ).

Conclusions GD is an independent risk factor of neonatal SRDS after $34^{0 / 7}$ weeks.

Keywords Gestational diabetes $\cdot$ Neonatal severe respiratory distress syndrome $\cdot$ Late preterm and term pregnancy $\cdot$ Maternal obesity

\section{Introduction}

Since its original description by O'Sullivan in 1961, the incidence of gestational diabetes (GD) has increased dramatically worldwide [1]. Evolution of alimentary habits, increase of obesity prevalence, and recent diminution of diagnostic threshold values contributed to this increase [2,3]. The incidence of GD nowadays reaches $17.8 \%$ worldwide, becoming a major public health concern [4]. Some materno-foetal complications of GD are now well-identified [5-11]. Thus, GD is known to expose to type 2 diabetes in post-partum [12] and to foetal consequences such as macrosomia, prematurity, and neonatal metabolic disorders such as severe neonatal hypoglycemia, hyperbilirubinemia, and hypocalcemia. Potential long-term and transgenerational effects have also been described [13]. Although an optimal balance of GD has been shown to significantly reduce neonatal morbidity 
and mortality [14], perinatal complications remain more frequent than in general population [10].

Respiratory distress syndrome occurs in a significant number of late preterm and term neonates and represents a major cause of admission in neonatal units with associated morbidity and mortality [15]. Aetiologies are multiple [16], with the two main causes being transient tachypnoea of the newborn related to abnormal lung fluid absorption and deficiency of surfactant. The risk of respiratory distress syndrome in relation with hyaline membrane disease in neonates from patients with GD has been previously reported [6]. However, the definition of respiratory distress syndrome throughout literature is not a homogeneous and the associated risk remains unclear after $34^{0 / 7}$ weeks of gestation (weeks). Although we previously identified GD as an independent risk factor for severe neonatal respiratory distress syndrome (SRDS) after $34^{0 / 7}$ weeks in a retrospective study with an aOR 11.55 (95\% CI 3.9-33.9, $p<0.001)$ [17] and two others retrospective studies gave contradictory conclusions in late-preterm infants from $34^{0 / 7}$ to $36^{6 / 7}$ weeks [18, 19], to our knowledge, no prospective study has yet demonstrated the association of SRDS and GD in late preterm and term neonates. The aim of this study is to prospectively assess if neonates delivered after $34^{0 / 7}$ weeks from mothers diagnosed with GD are exposed to an increased risk of neonatal SDRS.

\section{Methods}

\section{Patients}

We conducted a prospective cohort study among women who delivered in our tertiary care-level maternity unit in Marseille between January 1st 2011 and July 31st 2012. Women were recruited at the time of their admission in labour ward. All women with a monofoetal pregnancy in beginning of labour after $34^{0 / 7}$ weeks of gestation or planned for a caesarean section and who had been systematically screened for GD, according to our institution guidelines, were eligible to participate to the current study. Pregnant women with multiple pregnancy, existence of a pre-gestational diabetes, absence or poor pregnancy follow-up, uncertain dating of pregnancy, absence of systematic GD screening, and in utero foetal death were not included. The study protocol received approval from the Ethics Committee on Research in Obstetrics and Gynecology (CEROG no. 2010-OBS11-02). Written informed consent was obtained from all participants.

\section{Collected data}

Age, previous history, weight gain during pregnancy $(\mathrm{kg})$, body mass index (BMI) at the moment of delivery $\left(\mathrm{kg} / \mathrm{m}^{2}\right)$, and complications of pregnancy and time of occurrence were recorded for all participants. Maternal obesity was defined as $\mathrm{BMI} \geq 30 \mathrm{~kg} / \mathrm{m}^{2}$. We also recorded the exact term and mode of delivery (spontaneous vaginal delivery, assisted vaginal delivery, and caesarean section), the foetal gender, the neonatal weight, the Apgar score at $5 \mathrm{~min}$, and any diagnosed foetal malformation. Gestational age calculation was based on foetal biometry of cranio-caudal length at first-trimester systematic ultrasound scan performed between $11^{0 / 7}$ and $13^{6 / 7}$ weeks. Foetal growth restriction was defined as a neonatal weight lower than the 10th percentile and macrosomia as a neonatal weight over the 90 th percentile according to the reference curves from the AUDIPOG study [20,21]. The prenatal administration of corticosteroid therapy before $34^{0 / 7}$ weeks consisting in two intramuscular injections of $12 \mathrm{mg}$ of Betamethasone repeated over $24 \mathrm{~h}$ was also systematically recorded.

\section{Systematic screening of gestational diabetes}

According to our institution guidelines, screening of diabetes was systematically performed in all patients. Screening was performed using glucose tolerance test between $24^{0 / 7}$ and $28^{0 / 7}$ weeks independently of the existence of any identified maternal risk factors for GD. Overnight fasting plasma glucose was determined just before the administration of $75 \mathrm{~g}$ of glucose. Blood for glucose values was drawn at 1 and $2 \mathrm{~h}$. Diagnose of GD was made if at least one abnormal glucose value(s) was identified: glucose value after an overnight fast $\geq 0.92 \mathrm{~g} / \mathrm{L} ; 1$-h post-prandial level $\geq 1.80 \mathrm{~g} / \mathrm{L}$; and 2-h post-prandial level $\geq 1.53 \mathrm{~g} / \mathrm{L}$. In case of GD, the type of therapy (dietetic alone or associated with insulin therapy) and glucose level (6 capillary glycaemia per day) were monitored and documented. After 10 days of monitoring, controlled GD was defined by fast glucose values $<0.95 \mathrm{~g} / \mathrm{L}$ and post-prandial glucose values $<1.20 \mathrm{~g} / \mathrm{L}$.

\section{Neonatal outcomes and diagnosis of SRDS}

The primary outcome was the diagnostic of neonatal SRDS. As the definition of respiratory distress syndrome is quite heterogenic throughout literature, we chose to precisely define the SRDS using both clinical and radiological criteria. Thus, SRDS was defined by the association of clinical signs of early neonatal respiratory distress occurring within the first $2 \mathrm{~h}$ following birth, with consistent radiologic features and oxygen dependence requiring invasive and/or noninvasive mechanical ventilation with a fraction of inspired oxygen $(\mathrm{FiO} 2)>0.25$ for a minimum of $24 \mathrm{~h}$ and admission 
to neonatal intensive care unit (NICU). Duration of admission in NICU and of ventilation was systematically recorded.

\section{Number of subjects required and power of the study}

On a preliminary review of 300 deliveries from our institution, GD was diagnosed in $10.9 \%$ of women and was associated with $34.4 \%$ of SDRS in the GD group compared with $5.4 \%$ in control group ( $p<0.001)$ (unpublished data). Based on these results, the number of subjects required was calculated to detect a four-fold increased risk of SRDS in women with GD to achieve a $95 \%$ powered study with a $10 \%$ incidence of GD. We thus calculated the sample size to be at least of 429 women.

\section{Statistical analysis}

Statistical analysis was performed using the SPSS 17.0 software (SPSS Inc. Chicago, IL). Data were expressed as mean $\pm \mathrm{SE}$ or median (min-max). Groups were compared using the $\chi^{2}$ or Fisher exact test for categorical characteristics and using the Student $t$ test for continuous ones, as appropriate. A two-sided $p$ value $<0.05$ was considered as statistically significant.

\section{Results}

During the study period, a total of 444 pregnant women were included in the current study. GD was diagnosed in 60 patients $(13.5 \%)$.

\section{Maternal, obstetrical, and neonatal characteristics according to the diagnosis of gestational diabetes}

Patients and neonatal characteristics are summarized in Table 1 . The groups were statistically different in maternal age and maternal BMI at the time of delivery $(p<0.001)$.

Table 1 Maternal, obstetrical, and neonatal characteristics according to the diagnosis of maternal gestational diabetes

\begin{tabular}{|c|c|c|c|}
\hline & $\begin{array}{l}\text { Gestational diabetes } \\
(n=60)\end{array}$ & $\begin{array}{l}\text { No gestational diabetes } \\
(n=384)\end{array}$ & $p$ \\
\hline \multicolumn{4}{|l|}{ Maternal characteristics } \\
\hline Age (years) median (min-max) & $34(20-44)$ & $28(16-42)$ & $<0.001$ \\
\hline Smoking & $6(10)$ & $54(17)$ & 0.188 \\
\hline Nulliparity & $18(30)$ & $149(39)$ & 0.191 \\
\hline Obesity* & $35(66)$ & $115(35)$ & $<0.001$ \\
\hline \multicolumn{4}{|l|}{ Obstetrical characteristics } \\
\hline Gestational age at delivery (weeks) mean \pm SD & $39.3 \pm 1.6$ & $39.5 \pm 1.4$ & 0.31 \\
\hline Preterm birth** & $5(8)$ & $20(5)$ & 0.361 \\
\hline Corticosteroids therapy before $34^{0 / 7} \mathrm{WG}$ & $7(12)$ & $7(2)$ & $<0.001$ \\
\hline Induction of labour & $19(32)$ & $75(19)$ & 0.03 \\
\hline \multicolumn{4}{|l|}{ Mode of delivery } \\
\hline Spontaneous vaginal delivery & $33(55)$ & $263(68)$ & 0.061 \\
\hline Operative vaginal delivery & $5(8)$ & $38(10)$ & \\
\hline Planned c-section & $13(22)$ & $35(9)$ & \\
\hline Emergency c-section during labour & $9(15)$ & $48(12)$ & \\
\hline \multicolumn{4}{|l|}{ Neonatal outcomes } \\
\hline Neonatal weight mean \pm SD (grammes) & $3635.4 \pm 588.0$ & $3344.7 \pm 520.3$ & $<0.001$ \\
\hline Macrosomia*** & $13(23)$ & $39(10)$ & 0.026 \\
\hline Apgar $<7$ at $5 \mathrm{~min}$ & $7(12)$ & $6(2)$ & 0.001 \\
\hline Arterial umbilical $\mathrm{pH}$, mean $\pm \mathrm{SD}$ & $7.27 \pm 0.11$ & $7.29 \pm 0.08$ & 0.06 \\
\hline SRDS & $12(20)$ & $20(5)$ & $<0.001$ \\
\hline Length of hospitalization in NICU, median (min-max) (days) & $3(1-16)$ & $2(1-14)$ & 0.46 \\
\hline Length of mechanical ventilation, median (min-max) (days) & $1(0.04-15)$ & $1(0.02-4)$ & 0.11 \\
\hline
\end{tabular}

All values are expressed as $n(\%)$ otherwise specified

$S D$ standard deviation, $S R D S$ severe respiratory distress syndrome

* Body Mass index $>30\left(\mathrm{~kg} / \mathrm{m}^{2}\right)$

$* *<37$ weeks

*** Macrosomia defined as a neonatal weight $\geq 4000 \mathrm{~g}$ 
Compared to others, women diagnosed with GD were statistically older (mean age 32.5 years vs. 28.7 years, respectively, $p<0.001$ ), and significantly more likely to be obese at the moment of delivery ( $66 \%$ vs. $35 \%$, respectively, $p<0.001)$. Although mean gestational age at delivery was no statistically different between the two groups $(39.23 \pm 1.61$ in women with GD vs. $39.45 \pm 1.44$ in control group, $p=0.31$ ), women with GD were significantly more likely to have undergone induced labour $(p=0.03)$. When considering the different modes of delivery (spontaneous vaginal delivery, vaginal-assisted delivery, or caesarean section), no statistical difference was observed between the two groups.

Neonates delivered from women diagnosed with GD presented higher neonatal weight $(p=0.001)$ and higher rate of macrosomia $(p=0.02)$. A neonatal SRDS was diagnosed in 32 cases $(7.2 \%)$. No statistical difference was found in the median length of admission in NICU of neonates diagnosed with SRDS and in the median duration of mechanical ventilation. No case of neonatal death was recorded. Compared to others, neonatal SRDS was significantly more often observed in neonates from women diagnosed with GD
$12(20 \%)$ vs. $20(5.2 \%)$, respectively $(p<0.001)$. A higher proportion of neonates with an Apgar score $<7$ at 5 min was observed in neonates from women diagnosed with GD ( $p=0.001)$, but no statistical difference was found in the mean arterial umbilical $\mathrm{pH}(p=0.06)$.

\section{Maternal, obstetrical, and neonatal characteristics according to the occurrence of SRDS}

Maternal, obstetrical, and neonatal characteristics according to the occurrence of SRDS are summarized in Table 2. We found no statistically difference between the two groups regarding the following maternal characteristics: age, smoking status, nulliparity, and gestational age at delivery. GD was more frequently observed in women, whose neonates were diagnosed with SRDS 12 (38\%) vs. 48 (12\%), respectively $(p<0.001)$. Our study failed to identify any statistical difference in case of controlled GD $7(58.3 \%)$ vs. 35 $(72.9 \%)$, respectively $(p=0.481)$. Women whose neonates presented SRDS were more often obese: 18 (56\%) vs. 132 (32\%), respectively $(p=0.002)$. Furthermore, women whose neonates were diagnosed with SRDS were significantly more
Table 2 Maternal, obstetrical, and neonatal characteristics according to the occurrence of severe respiratory distress syndrome

\begin{tabular}{|c|c|c|c|c|}
\hline & \multicolumn{3}{|c|}{ Neonatal SRDS } & \multirow[t]{2}{*}{$p$} \\
\hline & Yes $(n=32)$ & & No $(n=412)$ & \\
\hline \multicolumn{5}{|l|}{ Maternal characteristics } \\
\hline Age median (min-max) (years) & $30.5(18-40)$ & $29(16-44)$ & & 0.882 \\
\hline Smoking & $6(19)$ & $64(16)$ & & 0.631 \\
\hline Nulliparity & $17(53)$ & $150(36)$ & & 0.060 \\
\hline Obesity* & $18(56)$ & $132(32)$ & & 0.002 \\
\hline Gestational diabetes & $12(38)$ & $48(12)$ & & $<.001$ \\
\hline \multicolumn{5}{|l|}{ Obstetrical characteristics } \\
\hline Gestational age at delivery mean $\pm \mathrm{SD}$ (weeks) & $38.9 \pm 2.1$ & $39.1 \pm 1.5$ & & 0.394 \\
\hline Preterm birth** & $3(9)$ & $22(5)$ & & 0.411 \\
\hline Maternal corticosteroids therapy before 34 weeks & $4(12)$ & $10(2)$ & & 0.013 \\
\hline Induction of labour & $10(31)$ & $83(20)$ & & 0.049 \\
\hline \multicolumn{5}{|l|}{ Mode of delivery $n(\%)$} \\
\hline Spontaneous vaginal delivery & $12(37)$ & $283(69)$ & & $<.001$ \\
\hline Operative vaginal delivery & $7(22)$ & $37(9)$ & & \\
\hline Planned c-section & $2(6)$ & $34(8)$ & & \\
\hline Emergency c-section during labour & $11(34)$ & $58(14)$ & & \\
\hline \multicolumn{5}{|l|}{ Neonatal outcomes } \\
\hline Neonatal weight, mean \pm SD (grammes) & $3465 \pm 696$ & $3375 \pm 523$ & & 0.378 \\
\hline Macrosomia*** & $4(13)$ & $48(12)$ & & 0.820 \\
\hline Apgar $<7$ at $5 \mathrm{~min}$ & $8(25)$ & $5(1)$ & & $<.001$ \\
\hline Arterial umbilical $\mathrm{pH}$, mean $\pm \mathrm{SD}$ & $7.21 \pm 0.13$ & $7.29 \pm 0.08$ & & $<.001$ \\
\hline
\end{tabular}

All values are expressed as $n(\%)$ otherwise specified

$S D$ standard deviation, $S R D S$ severe respiratory distress syndrome

* Body mass index $>30 \mathrm{~kg} / \mathrm{m}^{2}$

$* *<37$ weeks

*** Macrosomia defined as a neonatal weight $\geq 4000 \mathrm{~g}$ 
likely to have received corticosteroids therapy before $34^{0 / 7}$ weeks $4(12.5 \%)$ vs. $10(2.4 \%)$, respectively $(p=0.013)$.

\section{Mode of delivery and SRDS}

Compared to others, neonates diagnosed with SRDS were significantly more often born by caesarean section: 92 (22\%) vs. $13(40 \%)$, respectively ( $p<0.001)$. Caesarean sections were performed before $39^{0 / 7}$ weeks in 6 cases $(46 \%)$ in SRDS group versus in 19 (21\%) ( $p=0.07)$. Furthermore, neonates diagnosed with SRDS were significantly more often born after assisted vaginal delivery: 37 (9\%) vs. 7 $(22 \%)$, respectively $(p<0.001)$.

\section{Identification of independent risk factors for SRDS}

In multivariate analysis (Table 3), GD was identified as an independent risk factor of neonatal SRDS (aOR 3.6; 95\% CI 1.5-8.6; $p=0.005$ ). Other identified risk factors were obesity at time of delivery (aOR 2.8 ; $95 \%$ CI 1.1-7.1; $p=0.029$ ) and assisted vaginal delivery (aOR 5.5; CI 95\% $1.9-15.9 ; p=0.002$ ). Foetal macrosomia was not identified as an independent risk factor of neonatal SRDS.

\section{Discussion}

To our knowledge, this study is the first prospective study to report GD as an independent risk factor of neonatal SRDS after $34^{0 / 7}$ weeks of gestation. We believe these results to be of genuine importance in daily obstetrical practice as it might help for optimal neonatal care in anticipating the need for potential urgent neonatal management.

With a global rate up to $7 \%$ of SRDS after $34^{0 / 7}$ weeks of gestation, our results are consistent with those of the previous studies [22, 23]. Although using a rigorous definition of SRDS combining clinical and radiological criteria, the

Table 3 Identification of factor having a significant and independent impact on the risk of severe neonatal respiratory distress syndrome in multivariate logistic regression

\begin{tabular}{llll}
\hline & AOR & $95 \%$ CI & $p$ \\
\hline Obesity* & 2.8 & $1.1-7.1$ & 0.029 \\
Gestational diabetes & 3.6 & $1.5-8.6$ & 0.005 \\
Planned c-section & 1.9 & $0.6-6.0$ & 0.284 \\
Emergency c-section during labour & 2.9 & $0.9-9.2$ & 0.071 \\
Operative vaginal delivery & 5.5 & $1.9-15.9$ & 0.002 \\
Macrosomia** $^{*}$ & 0.9 & $0.3-2.9$ & 0.848 \\
\hline
\end{tabular}

$C I$ confidence interval, $A O R$ adjusted odds ratio

* BMI > $30\left(\mathrm{~kg} / \mathrm{m}^{2}\right)$

** Defined as a neonatal weight $\geq 4000 \mathrm{~g}$ prevalence of SRDS reached up to $20 \%$ of neonates from patients diagnosed with GD. It is noticeable that, differently from the previous studies, we did not observe any statistical association between the diagnosis of foetal macrosomia and the occurrence of SRDS [24-26]. Because macrosomia is known as a major complications of GD and controlled GD was demonstrated to be associated with a diminution of the incidence of macrosomia [27, 28], we can hypothesize our results to be explained by the small size of our study. Furthermore, we identified operative vaginal delivery as an independent risk factor of SRDS. We believe this result to be easily explained by the fact that more than the half of operative vaginal deliveries was indicated by non-reassuring foetal heart rate, being the genuine cause of poor neonatal outcome. Caesarean delivery even planned or during labour was not associated with SDRS in multivariate logistic regression contrary to results reported in a recent retrospective study of late-preterm births [19] and lack of power or different period of inclusion (late preterm and term) can explain this difference.

The direct effect of maternal GD on the risk of neonatal SRDS may have physiopathological and clinical explanations. First, GD has been experimentally shown to induce both insufficient and an abnormal maturation of surfactant due to immaturity of type II alveolar cells from rats, with particularly important effects at the end of pregnancy [29]. Furthermore, phosphatidyl glycerol, an essential component of surfactant, may be diminished, due to a delayed secretion in case of unbalanced GD [30]. Second, induced labour or planned caesarean section is more likely to be performed in cases of GD, independently of suspected macrosomia, resulting in an increased risk of neonatal respiratory distress syndrome due to a delayed resorption of pulmonary liquid or "wet lung" [31-33]. Our study reported that women diagnosed with GD were more likely to have undergone induced labour and neonates diagnosed with SRDS were more often born after the induction of labour. Finally, GD is known to be associated with an increased risk of prematurity. A prospective cohort study on 46,230 pregnancies reported this risk to depend on the levels of maternal glycemia, independently of perinatal complications that could have triggered early delivery such as gestational hypertension, preeclampsia, hydramnios, or macrosomia [34]. Another study comforted these results in women with impaired glucose tolerance [35]. We found in our study a trend of increasing risk of preterm birth in women with GD but our study failed to demonstrate a significant difference, possibly because of a lack of power, with small effectives in each group.

We found obesity to be an independent risk factor of SRDS. This finding is consistent with the previous literature as maternal obesity has already been shown to be associated with a significantly higher risk of perinatal death [36]. Obesity in women with GD has also been documented to 
be associated with a higher proportion of adverse neonatal outcomes, including the need respiratory support [37]. Our study failed to identify a benefit in the achievement of optimal glycaemic level during pregnancy. Such optimal balance of glucose level by insulin therapy has, however, been previously shown to be the sole factor associated with a reduction of neonatal complications in women with GD $[14,38]$. Although early optimal balance of GD seems to be crucial to reduce neonatal complications, the association of controlled GD and of decreasing risk of SRDS remains undemonstrated. However, the small effectives in each group and a non-dedicated design of our study do not allow for any genuine conclusion and further large prospective studies remain needed to properly answer to that question.

Our results raise concerns regarding the potential benefit of corticosteroids therapy performed between $34^{0 / 7}$ and $36^{6 / 7}$ weeks in case of GD with the risk of imminent delivery to reduce the risk of SRDS. This question was asked in a retrospective study with a higher proportion of infants from the GD group receiving corticosteroids but without impact on logistic regression analysis [18]. A randomized trial recently suggested that the administration of betamethasone to women at risk for late-preterm delivery (from $34^{0 / 7}$ to $36^{5 / 7}$ weeks) could significantly reduce the rate of neonatal respiratory related morbidity [39]. In this trial, the proportion of women with GD was the same in both betamethasone and placebo groups (around 10\%). It is, however, noticeable that the primary outcome was a neonatal composite of treatment in the first $72 \mathrm{~h}$ and did not specifically describe the severity of SRDS. Although that outcome decreased from $14.4 \%$ (placebo) to $11.6 \%$ (betamethasone), the incidence of neonatal hypoglycaemia was greatly increased in the steroid group. At this stage, the benefit of corticosteroids therapy in patients with GD and threatened late-preterm delivery remains, however, questionable; more studies are needed to investigate this point.

Finally, in showing GD to be an independent risk factor of neonatal SRDS after $34^{0 / 7}$ weeks of gestation, our results suggest the need of reorganizing the management of pregnant women diagnosed with GD and to decide, where these patients should deliver, especially since the incidence of GD reaches $13.5 \%$ in our study. This incidence is lower than reported in the French national perinatal surveys in 2010 (7.2\%) in general population but concordant with incidence in high risk population in France (10-20\%) [40]. However, this incidence is lower than the worldwide incidence probably because of difference in incidence of obesity, maternal age, ethnicity, and other factors influencing the occurrence of GD. As recently highlighted by the European Consensus Guidelines on the Management of Respiratory Distress Syndrome [41], "optimizing the outcome for babies with respiratory distress syndrome includes good obstetric practice and consideration of whether transfer to a perinatal centre is necessary and safe". Nowadays, GD still does not justify management in a specific level of care maternity unit. Most will deliver in low level of care maternity units, with paediatrician not being systematically present at the time of delivery. Our results bring additional arguments for a genuine reflection about these recommendations.

\section{Conclusion}

GD is an independent risk factor of neonatal SRDS after $34^{0 / 7}$ weeks. This risk is also increased in obese women. These results confirm the need for careful GD screening during pregnancy, with antenatal prevention of maternal obesity. They also suggest the need for anticipation of the management of pregnant women diagnosed with GD and specially to transfer them in perinatal centre with the possibility of systematic immediate neonatal paediatric management if required.

Author contributions MI: data collection and manuscript writing, BJ: manuscript writing, TB: reviewing, GC: supervision and methodology, BF: supervision and methodology, CX: Project development, reviewing, and supervision.

\section{Compliance with ethical standards}

Conflict of interest The authors declare that they have no conflict of interest.

Ethical approval All procedures performed in this study were in accordance with the ethical standards of the national research committee and the study protocol received approval from the Ethics Committee on Research in Obstetrics and Gynecology (CEROG no. 2010OBS11-02).

Informed consent Written informed consent was obtained from all participants included in the study.

\section{References}

1. O'sullivan JB (1961) Gestational diabetes. Unsuspected, asymptomatic diabetes in pregnancy. N Engl J Med 264:1082-1085

2. Leary J, Pettitt DJ, Jovanovic L (2010) Gestational diabetes guidelines in a HAPO world. Best Pract Res Clin Endocrinol Metab 24(4):673-685

3. International Association of Diabetes and Pregnancy Study Groups Consensus Panel, Metzger BE, Gabbe SG, Persson B, Buchanan TA, Catalano PA et al (2010) International association of diabetes and pregnancy study groups recommendations on the diagnosis and classification of hyperglycemia in pregnancy. Diabetes Care 33(3):676-682

4. Legardeur H, Girard G, Mandelbrot L (2011) Screening of gestational diabetes mellitus: a new consensus? Gynecol Obstet Fertil 39(3):174-179

5. Hurwitz D, Higano N (1952) Diabetes and pregnancy. N Engl J Med 247(9):305-309 
6. Robert MF, Neff RK, Hubbell JP, Taeusch HW, Avery ME (1976) Association between maternal diabetes and the respiratory-distress syndrome in the newborn. N Engl J Med 294(7):357-360

7. Schwartz R, Teramo KA (2000) Effects of diabetic pregnancy on the fetus and newborn. Semin Perinatol 24(2):120-135

8. Svare JA, Hansen BB, Mølsted-Pedersen L (2001) Perinatal complications in women with gestational diabetes mellitus. Acta Obstet Gynecol Scand 80(10):899-904

9. Gilmartin ABH, Ural SH, Repke JT (2008) Gestational diabetes mellitus. Rev Obstet Gynecol 1(3):129-134

10. Weindling AM (2009) Offspring of diabetic pregnancy: short-term outcomes. Semin Fetal Neonatal Med 14(2):111-118

11. Mitanchez D (2010) Fetal and neonatal complications of gestational diabetes: perinatal mortality, congenital malformations, macrosomia, shoulder dystocia, birth injuries, neonatal outcomes. J Gynécol Obstét Biol Reprod 39(8 Suppl 2):S189-S199

12. Bellamy L, Casas J-P, Hingorani AD, Williams D (2009) Type 2 diabetes mellitus after gestational diabetes: a systematic review and meta-analysis. Lancet Lond Engl 373(9677):1773-1779

13. Mitanchez D, Burguet A, Simeoni U (2014) Infants born to mothers with gestational diabetes mellitus: mild neonatal effects, a long-term threat to global health. J Pediatr 164(3):445-450

14. Crowther CA, Hiller JE, Moss JR, McPhee AJ, Jeffries WS, Robinson JS et al (2005) Effect of treatment of gestational diabetes mellitus on pregnancy outcomes. N Engl J Med 352(24):2477-2486

15. Edwards MO, Kotecha SJ, Kotecha S (2013) Respiratory distress of the term newborn infant. Paediatr Respir Rev 14(1):29-36 (quiz 36-37)

16. Kjos SL, Walther FJ, Montoro M, Paul RH, Diaz F, Stabler M (1990) Prevalence and etiology of respiratory distress in infants of diabetic mothers: predictive value of fetal lung maturation tests. Am J Obstet Gynecol 163(3):898-903

17. Vignoles P, Gire C, Mancini J, Bretelle F, Boubli L, Janky E et al (2011) Gestational diabetes: a strong independent risk factor for severe neonatal respiratory failure after 34 weeks. Arch Gynecol Obstet 284(5):1099-1104

18. Fung GPG, Chan LM, Ho YC, To WK, Chan HB, Lao TT (2014) Does gestational diabetes mellitus affect respiratory outcome in late-preterm infants? Early Hum Dev 90(9):527-530

19. Bricelj K, Tul N, Lucovnik M, Kronhauser-Cerar L, Steblovnik L, Verdenik I et al (2017) Neonatal respiratory morbidity in late-preterm births in pregnancies with and without gestational diabetes mellitus. J Matern-Fetal Neonatal Med Off J Eur Assoc Perinat Med Fed Asia Ocean Perinat Soc Int Soc Perinat Obstet 30(4):377-379

20. Mamelle N, Munoz F, Grandjean H (1996) Fetal growth from the AUDIPOG study. I. Establishment of reference curves. J Gynecol Obstet Biol Reprod (Paris) 25(1):61-70

21. Mamelle N, Munoz F, Martin JL, Laumon B, Grandjean H (1996) Fetal growth from the AUDIPOG study. II. Application for the diagnosis of intrauterine growth retardation. J Gynecol Obstet Biol Reprod (Paris) 25(1):71-77

22. Dani C, Reali MF, Bertini G, Wiechmann L, Spagnolo A, Tangucci M et al (1999) Risk factors for the development of respiratory distress syndrome and transient tachypnoea in newborn infants. Italian Group of Neonatal Pneumology. Eur Respir J 14(1):155-159

23. Kumar A, Bhat BV (1996) Epidemiology of respiratory distress of newborns. Indian J Pediatr 63(1):93-98
24. Esakoff TF, Cheng YW, Sparks TN, Caughey AB (2009) The association between birthweight $4000 \mathrm{~g}$ or greater and perinatal outcomes in patients with and without gestational diabetes mellitus. Am J Obstet Gynecol 200(6):672.e1-672.e4

25. HAPO Study Cooperative Research Group (2009) Hyperglycemia and adverse pregnancy outcome (HAPO) study: associations with neonatal anthropometrics. Diabetes 58(2):453-459

26. Das S, Irigoyen M, Patterson MB, Salvador A, Schutzman DL (2009) Neonatal outcomes of macrosomic births in diabetic and non-diabetic women. Arch Dis Child Fetal Neonatal Ed 94(6):F419-F422

27. Langer O, Yogev Y, Most O, Xenakis EMJ (2005) Gestational diabetes: the consequences of not treating. Am J Obstet Gynecol 192(4):989-997

28. Horvath K, Koch K, Jeitler K, Matyas E, Bender R, Bastian H et al (2010) Effects of treatment in women with gestational diabetes mellitus: systematic review and meta-analysis. BMJ 1(340):c1395

29. Gewolb IH, O'Brien J (1997) Surfactant secretion by type II pneumocytes is inhibited by high glucose concentrations. Exp Lung Res 23(3):245-255

30. Piper JM (2002) Lung maturation in diabetes in pregnancy: if and when to test. Semin Perinatol 26(3):206-209

31. Ehrenberg HM, Durnwald CP, Catalano P, Mercer BM (2004) The influence of obesity and diabetes on the risk of cesarean delivery. Am J Obstet Gynecol 191(3):969-974

32. Al-Agha R, Kinsley BT, Finucane FM, Murray S, Daly S, Foley $M$ et al (2010) Caesarean section and macrosomia increase transient tachypnoea of the newborn in type 1 diabetes pregnancies. Diabetes Res Clin Pract 89(3):e46-e48

33. Al-Qahtani S, Heath A, Quenby S, Dawood F, Floyd R, Burdyga $T$ et al (2012) Diabetes is associated with impairment of uterine contractility and high Caesarean section rate. Diabetologia 55(2):489-498

34. Hedderson MM, Ferrara A, Sacks DA (2003) Gestational diabetes mellitus and lesser degrees of pregnancy hyperglycemia: association with increased risk of spontaneous preterm birth. Obstet Gynecol 102(4):850-856

35. Ostlund I, Hanson U, Björklund A, Hjertberg R, Eva N, Nordlander E et al (2003) Maternal and fetal outcomes if gestational impaired glucose tolerance is not treated. Diabetes Care 26(7):2107-2111

36. Chu SY, Kim SY, Lau J, Schmid CH, Dietz PM, Callaghan WM et al (2007) Maternal obesity and risk of stillbirth: a metaanalysis. Am J Obstet Gynecol 197(3):223-228

37. Langer O, Yogev Y, Xenakis EMJ, Brustman L (2005) Overweight and obese in gestational diabetes: the impact on pregnancy outcome. Am J Obstet Gynecol 192(6):1768-1776

38. Landon MB, Spong CY, Thom E, Carpenter MW, Ramin SM, Casey B et al (2009) A multicenter, randomized trial of treatment for mild gestational diabetes. N Engl J Med 361(14):1339-1348

39. Gyamfi-Bannerman C, Thom EA (2016) Antenatal betamethasone for women at risk for late preterm delivery. N Engl J Med 375(5):486-487

40. Galtier F (2010) Definitions, epidemiology, risk factors. J Gynecol Obstet Biol Reprod (Paris) 39(8 Suppl 2):S144-S170

41. Sweet DG, Carnielli V, Greisen G, Hallman M, Ozek E, Plavka R et al (2016) European consensus guidelines on the management of respiratory distress syndrome-2016 update. Neonatology 111(2):107-125 